\title{
WHITE DWARF COLORS IN LOW ACCRETION RATE BINARIES
}

\author{
DEAN M. TOWNSLEY \\ Department of Physics, University of California, Santa Barbara \\ Broida Hall, Santa Barbara, CA 93106 \\ AND \\ LARS BILDSTEN \\ Kavli Institute for Theoretical Physics and Department of Physics, \\ University of California, Santa Barbara \\ Kohn Hall, Santa Barbara, CA 93106
}

\begin{abstract}
.
Our recent theoretical work (Townsley and Bildsten 2002) on the thermal state of white dwarfs (WDs) in low mass transfer rate binaries allows us to predict the broadband colors of the binary from those of the WD and companion when the disk is dim. The results based on standard CV evolution are presented here. These will aid the discovery of such objects in field surveys and proper-motion selected globular cluster surveys with HST; especially for the largely unexplored post period minimum Cataclysmic Variables $(\mathrm{CVs})$ with the lowest accretion rates and degenerate companions. We have also calculated the fraction of time that the WD resides in the ZZ Ceti instability strip thus clarifying that we expect many accreting WDs to exhibit non-radial oscillations. The study of these will provide new insights into the rotational and thermal structure of an actively accreting WD.
\end{abstract}

\section{Introduction}

As summarized elsewhere in these proceedings our recent work has demonstrated that at the low accretion rates appropriate for dwarf novae (DN), the core temperature, $T_{c}$, of the WD is set by the long-time average accretion rate, $\langle\dot{M}\rangle$, of the binary [1]. With $T_{c}$ set, we determine the WD luminosity, which depends on $\langle\dot{M}\rangle, M_{\mathrm{WD}}$, and the mass of the freshly ac- 


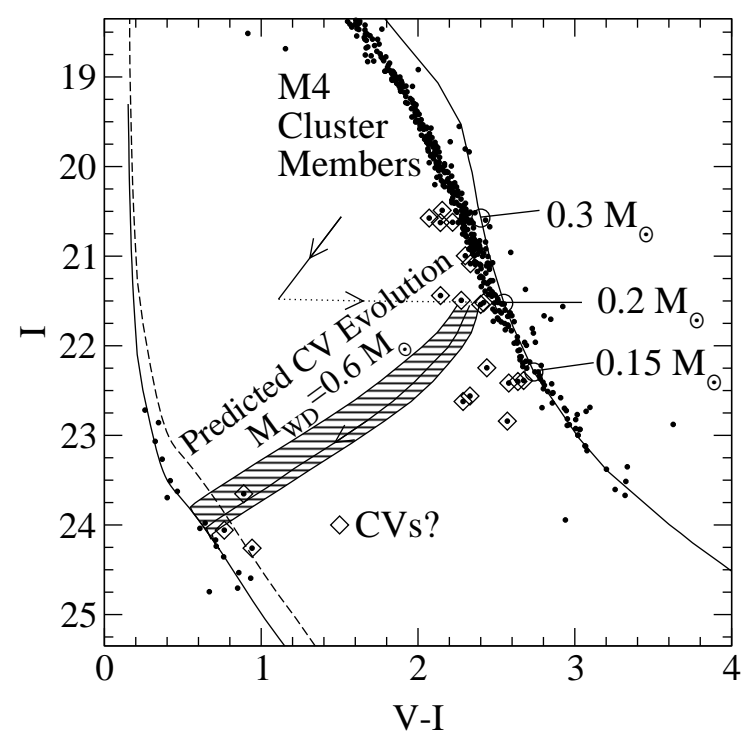

Figure 1. Evolutionary path for a $0.6 M_{\odot}$ WD in a CV. For simplicity, only contributions from the WD and MS companion have been included. The displayed HST data are proper motion selected cluster members [4].

creted layer. This luminosity is directly observable for DN when the disk is in quiescence and the surface of the WD has cooled after outburst.

\section{Results}

In the quiescent state, the broadband colors are just that of a hot WD plus a low mass main sequence star or brown dwarf companion. Figure 1 shows the color and magnitude we predict for quiescent CV binaries using standard CV evolution to relate the companion mass to $\langle\dot{M}\rangle[2,3]$. The data shown for comparison are members of the globular cluster M4 as determined by proper motion studies with $H S T$ [4]. The $0.6 M_{\odot}$ WD primary spends approximately 1 Gyr in the ZZ Ceti instability strip.

This work was supported by the NSF under Grants PHY99-07949, AST01-96422, and AST02-05956, and by NASA through grant AR-09517.01A from STScI, which is operated by AURA, Inc., under NASA contract NAS5-26555. L. B. is a Cottrell Scholar of the Research Corporation and D. T. is an NSF Graduate Fellow.

\section{References}

1. Townsley, D. M. \& Bildsten, L. 2002, ApJ, 565, L35

2. Howell, S. B., Nelson, L. A., \& Rappaport, S. 2001, ApJ, 550, 897

3. Kolb, U. \& Baraffe, I. 1999, MNRAS, 309, 1034

4. King, I. R., Anderson, J., Cool, A. M., \& Piotto, G. 1998, ApJ, 492, L37 